\title{
Effect of Pre-Treatment on the Morphology and Chemical Properties of Polyethylene Terephthalate (PET)/Pineapple Leaf Fiber (PALF) Electrospun Mat
}

\author{
S N Surip ${ }^{1}$, F M A Aziz ${ }^{1 *}$, A H Yuwono², N Sofyan ${ }^{2}$ \\ ${ }^{1}$ Faculty of Applied Sciences, Universiti Teknologi MARA, 40450 Shah Alam, Selangor, Malaysia \\ ${ }^{2}$ Department of Metallurgical and Materials Engineering, Faculty of Engineering, Universitas Indonesia, 16424 Depok Indonesia \\ *Corresponding author E-mail: fatimahmuyassarah@gmail.com
}

\begin{abstract}
In the past decades, conventional petroleum-based plastics have resulted in environmental and sustainability issues. Thus, there has been significant interest in the utilization of natural materials for nanofibers product such as for filtration media. However, poor compatibility exists between polymers and natural fibers due to natural fibers hydrophilic properties leading to poor nanofibers formation. In this study, Pineapple Leaf Fiber (PALF) remarkable properties were explored. PALF undergo alkaline treatment and bleaching treatment in order to improve its compatibility. Thermal, morphology and structural properties of PALF raw (PR), PALF after alkali treatments (PA) and PALF after alkali + bleaching treatment (PB) were studied. Further, all the samples were diluted using Trifluoroacetic Acid (TFA) as the solvent and Polyethylene Terephthalate (PET) as the polymer carrier and proceed to electrospinning to produce a nanofibers electrospun mats. The electrospun mats were then characterized in terms of its chemical properties using Fourier transform infrared spectroscopy (FTIR) as well as the morphology which using Fields Emission Scanning Electron Microscopy (FESEM). FTIR result shows the electrospun PET does not produce any peak at $\sim 3400 \mathrm{~cm}-1$ due to its hydrophobic properties. Nevertheless, with addition of PALF, the peak was significantly increased. FESEM results indicated that the present of fibers led to a tendency of lower average fiber diameter compared to the neat PET. Unconnected and thin fibers were coexited from single fiber of PALF raw electrospun indicated that new fibers were ejected however the bond were collapsed during ejection thus did not produce a complete single fiber. Despite that, more uniform fibers of electrospun mat were produced by pre-treatment of PALF.
\end{abstract}

Keywords: Pineapple Leaf Fiber(PALF), Pre-treatments, Polyethylene Terephthalate (PET), Electrospinning, Nanofibers.

\section{Introduction}

Recently, researchers start to develop interest on green and sustainable materials to replace synthetic fibers due to the critical drawbacks of synthetic fibers [1]. Natural fibers were claimed to offer environmental advantages such as renewable, biodegradable, green friendly and good mechanical properties [2]. Natural fiber such as sisal fibers, bamboo fibers and pineapple leaf fibers that possesses strong mechanical properties had been used long ago for housework purposes such as for ropes and rattan basket. As time changes, natural fibers have become highly demands in industrial market with varies of applications such as for medical field, industrial and textile [4].

Pineapple leaf fibers (PALF) is one of natural fibers that possess great mechanical properties which still unexplored widely. Pineapple plant is referred as secondary plant which by-product wastes were extracts to obtain cellulose. According to Neto et al (2015), cellulose content act directly proportional with mechanical properties while lignin vice versa. PALF consists of high amount of cellulose $(70 \%-82 \%)$ and low lignin content $(5-12 \%)$ thus made it suitable for industrial purposes [5]. However, lack of good interfacial adhesion, low melting point, and poor resistance towards moisture make the use of PALF less attractive. Pretreatments of the natural fibers can help to improve the performance. Pre- treatment were done in two methods, mechanical or chemical treatment [6].

Among the various pretreatment techniques, chemical treatment was common treatment used for natural fibers [7]. PALF is organized into fibrils, which are surrounded by a matrix of lignin, hemicellulose and pectin. Hemicelluloses were present and located within the structure of cellulose. The strong bond between hemicellulose and cellulose were believed to decrease the average crystallinity of the cellulose fibrils [2]. Reducing lignin and hemicellulose amount help to increased crystalline cellulose content which contributed to improvement on thermal and chemical properties [1]. Nadezda et al (2016) in the studied proved that the thermal stability of wood pulp fibers improved after bleaching treatment thus provide materials with great heat resistant.

However, even there are many papers already been published on PALF, there were only few that were proceed to the PALF nanofibers. Nanofiber materials had a good catch up with industrial requirement due to its large surface area, superior mechanical performances and varieties of surface functionalities [8]. Electrospinning method is a convenient with a multi-purpose method that aims on producing fibers with diameter range from several micrometers to tens of nanometers [9]. Through this method, nanofibers size of PALF could be produced. Recently, Guihe and the co-workers succeed in produced mats of electrospun PET/PVA with a diameter range $200 \mu \mathrm{m}$ to $700 \mu \mathrm{m}$ with higher mechanical properties when compared to pure PET [10]. 
Electrospun nanofibers have become promising materials for many biomedical applications such as wound dressing, drug delivery and tissue scaffold $[11,12]$. Besides, it is also known for industrial purposes such as for reinforcing materials, filtration media and protective clothing [13].

\section{Experimental}

\subsection{Materials}

Pineapple Leaf fibers were received from local Malaysian company in fibers form. Polyethylene Terephthalate were obtained from Aldrich and received in granular form. Chemicals which are Trifluoroacetic acid (TFA), Hydrogen Peroxide $\left(\mathrm{H}_{2} \mathrm{O}_{2}\right)$, Hydrochloric acid (HCL), Sodium Hydroxide $(\mathrm{NaOH})$, Toluene and Ethanol all were supplied by Aldrich Merck.

\subsection{Methods}

\subsubsection{Dewaxing}

PALF were first extracted with toluene/ethanol $(2: 1 \mathrm{v} / \mathrm{v})$ for 6 hours in order to remove organic compounds from the surface thus increase the reaction.

\subsubsection{Chemical Treatment of PALF}

PALF was treated with $2 \mathrm{M}$ of Hydrochloric Acid (HCL) at $80^{\circ} \mathrm{C}$ for 2 hours. Later, the sample was treated using $2 \mathrm{M}$ of $\mathrm{NaOH}$ at $80^{\circ} \mathrm{C}$ for 2 hours (alkali treatment). Lastly, the sample were soaked with $\mathrm{H}_{2} \mathrm{O}_{2}$ (bleaching) for 5 hours to reduce amount of lignin in the sample. Figure 1 shows sample of raw PALF and PALF after treatment. Samples were labelled as PR, PA and PB respectively as shown in figure 1 .

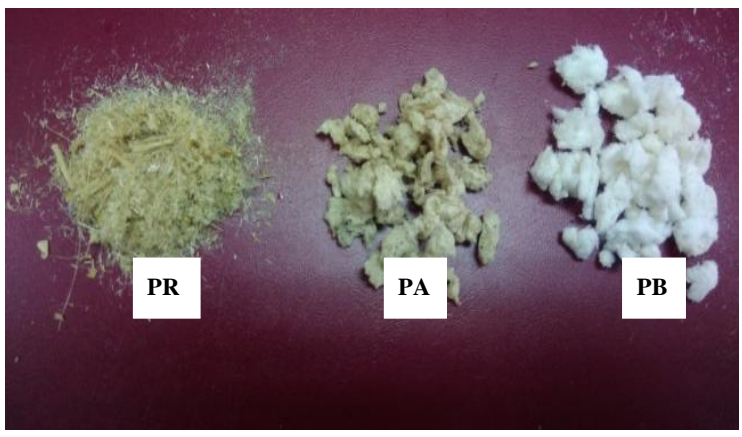

Fig. 1: (PR) Raw PALF (PA) Alkali Treatment PALF (PB) Alkali + Bleaching Treatment PALF

\subsubsection{PALF solution preparation}

The samples were diluted using Trifluoroacetic Acid (TFA) as the solvent and Polyethylene Terephthalate (PET) as the polymer carrier and proceed to electrospinning to produce a nanofibers electrospun mats. PALF and PET were dissolved in TFA to obtain $2 \times 10 \mathrm{~g} \mathrm{~mL}^{-1}$ solution concentration (9). The samples were stirred for 6 hours at room temperature until the solution become homogenous.

\subsubsection{Electrospinning}

The electrospinning solutions were placed into a $3 \mathrm{~mL}$ syringe with a metallic needle diameter $0.3 \mathrm{~mm}$ which connected to a pump with $0.5 \mathrm{ml} / \mathrm{h}$ flow rate. A copper wire connected to the positive charge was inserted into solution. Fibers were collected as a nonwoven fibrous mat by aluminium foil wrapped collecter that were placed $12 \mathrm{~cm}$ from the needle tip. Figure 2 shows the electrospinning machine set up.

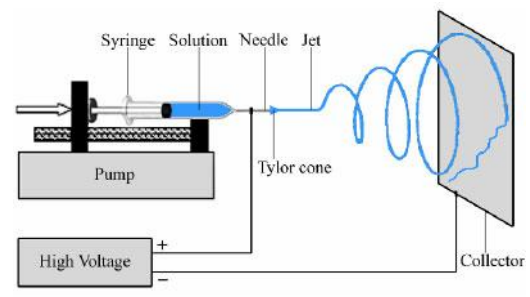

Fig. 2: Electrospinning machine set up

\subsection{Characterization}

\subsubsection{FTIR}

FTIR spectroscopy was carried out in order to study the interaction that occurred in TFA and PALF. This kind of interaction can be proven through frequency shifts, changes in band intensity, and shape of the FTIR spectra. The spectra were recorded by FTIR (Thermo Fisher Scientific Nicolet iS 10) equipped with Attenuated Total Reflectance (ATR) in the transmittance mode over a frequency range $500-4000 \mathrm{~cm}-1$ with $4 \mathrm{~cm}^{-1}$ resolutions.

\subsubsection{STA}

Simultaneous Thermal Analysis TGA-DTA/DSC measures both heat flow (Differential Scanning Calorimetry) and weight changes (thermogravimetry) in a material as a function of temperature or time in a controlled atmosphere. STA were performed using (Perkin Elmer STA 600) and conducted in a temperature range $30^{\circ} \mathrm{C}-800^{\circ} \mathrm{C}$ with heating rate $10^{\circ} \mathrm{C} / \mathrm{min}$. The analysis was carried out under nitrogen atmosphere at a flow rate of $20 \mathrm{~mL}-\mathrm{min}^{-1}$.

\subsubsection{FESEM}

Sample morphology was observed under FESEM at an accelerating volt of $5.0 \mathrm{kV}$. All samples were coated with a thin layer of gold to reduce charging and produce a conductive surface. The diameters were calculated using Microsoft word.

\section{Result \& Discussion}

\subsection{Effect of pre-treatment on the chemical structure of PALF}

Figure 3 (a) shows the FTIR spectra of PR, PA and PB respectively. The peak observed at approximately $\sim 3300 \mathrm{~cm}^{-1}$ that represents $\mathrm{O}-\mathrm{H}$ bonding comes in the presence of carbohydrate (cellulose + hemicelluloses) and lignin [14]. The peak intensities were increased after each treatment suggested that this is due to the broken hydroxyl group which is alkaline sensitive thus promotes free hydroxyl that caused the addition of extra peak in free hydroxyl bond structure at $2823 \mathrm{~cm}^{-1}$ [15].

Figure 3 (b) illustrates two peaks at $1725 \mathrm{~cm}^{-1}$ and $1650 \mathrm{~cm}^{-1}$ that represent $\mathrm{C}=\mathrm{O}$ (hemicelluloses) and lignin (aromatic $\mathrm{C}=\mathrm{C}$ ) respectively. At $1725 \mathrm{~cm}^{-1}$ region, hemicelluloses were completely removed after alkali treatment (PA). This happens because hemicellulose become easily hydrolysed in alkaline medium due to the breaking $\mathrm{C}-\mathrm{O}-\mathrm{C}$ bond [17]. Indeed, alkaline treatment is known as effective method to remove hemicelluloses $[7,14]$. At $1650 \mathrm{~cm}^{-1}$ region which represent lignin, PA peak shift to lower wavenumber indicating weak interaction between the $\mathrm{C}=\mathrm{C}$ bond. Nevertheless, no significant change in the intensity of the peak were observed. The strong aromatic structure of lignin makes it hard to be removed from the fibers [18].

The peaks at $1373 \mathrm{~cm}^{-1}$ and $667 \mathrm{~cm}^{-1}$ represent the $\mathrm{C}-\mathrm{H}$ bond of cellulose were observed in Figure 4 (b). No significant changes were observed in the intensity of $1373 \mathrm{~cm}^{-1}$. However, at peak $667 \mathrm{~cm}^{-1}$, peak intensity for PA and PB were increased and the peak shifts to higher wavenumber which is $670 \mathrm{~cm}^{-1}$ and $673 \mathrm{~cm}^{-1}$ 
respectively. According to M. Le Troedec et al (2008), crystalline cellulose of fiber can be calculated from ratio of the band at $1373 \mathrm{~cm}^{-1}$ and $667 \mathrm{~cm}^{-1}[18]$. Treatment fibers shows high crystalline cellulose contributed to thermal properties enhancement. Despite that, peak that come from raw PALF at $1250 \mathrm{~cm}^{-1}$ that represent pectin and lignin, were completely removed after alkali treatment indicating removal of pectin during treatment. Removal of hemicellulose, pectin and waxes lead to the rearrangement of crystalline cellulose chains in a particular order that contributed to strong mechanical properties [7]. Furthermore, at region $1030 \mathrm{~cm}^{-1}$ to $1060 \mathrm{~cm}^{-1}$, single peak of PR has become combinations of two different small peak of $1037 \mathrm{~cm}^{-1}$ and $2053 \mathrm{~cm}^{-1}$ respectively after alkali and bleaching treatment. Cao et al suggest that both of this peak come from $\mathrm{C}-\mathrm{O}$ stretching bond of functional group of glycoside linkage.

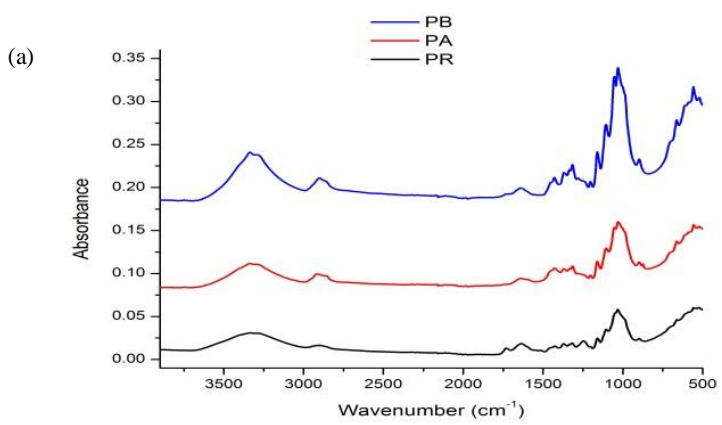

(b)

(c)
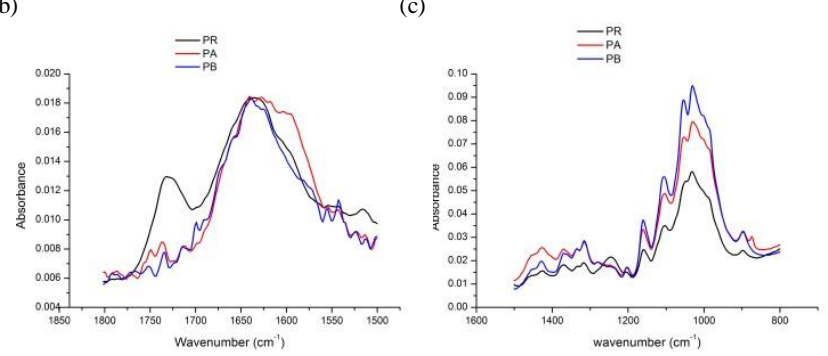

Fig. 3: (a) FTIR spectra of PR, PA and PB (b) zoom in at $1500-1800 \mathrm{~cm}^{-1}$ (c) zoom in at $800-1500 \mathrm{~cm}^{-1}$

\subsection{Effect of pre-treatment on thermal properties (TGA) of PALF}

Figure 4(a) and 4 (b) shows TGA and DTG results for PR, PA and PB respectively. TGA result (Figure 4(a)) shows that initial weight losses were observed at temperature $20^{\circ} \mathrm{C}-100{ }^{\circ} \mathrm{C}$ resulting from moisture evaporation. This common loss mass depends on the initial moisture content of the fibers. PR shows lower moisture content, 2.3\% compared to PA and PB which were 5.4\% and 7.6\% respectively. Second stage loss for PR occurred at temperature between $282{ }^{\circ} \mathrm{C}$ to $367^{\circ} \mathrm{C}$ and likely contributed by degradation of hemicellulose and initial broken glycosidic linkages of cellulose [2]. Nevertheless, this stage lose were completely absent for PA and $\mathrm{PB}$ indicating completely removal of hemicellulose during treatment. Low thermal stability of hemicellulose made it easily degraded before cellulose and lignin. According to Asim et al (2016), thermal stability of a fiber was determined using initial degradation temperature of hemicellulose.

Weight losses for PR, PA and PB at temperature $367{ }^{\circ} \mathrm{C}-415{ }^{\circ} \mathrm{C}$ are $27 \%, 39 \%$ and $47 \%$ respectively. These results are indicating of a fast rate of fiber degradation and increased with treatment PALF. PR shows low percentage mass loss compared to PA and PB. This is because raw PALF (PR) cellulose was surrounded by lignin, hemicellulose and pectin. Hemicellulose were strongly connected to the cellulose thus is believed to decreased the average crystallinity of cellulose [2]. At $415{ }^{\circ} \mathrm{C}$ to $600{ }^{\circ} \mathrm{C}$, only $4.3 \%$ $5.0 \%$ lignin weight losses were observed. This small percentage loss occurs due to broken of lignin and formation of aromatic in nitrogen atmosphere [14]. There is also a distinction between the amounts of the residue of PALF which is $14.0 \%, 13.26 \%$ and $7.52 \%$

In figure 4 (b), it can be seen that PALF has become slightly hydrophobic after treatment as the water losses increased. Besides, the moisture loss peak shifted to higher temperature for PB indicated improvement of structure of water that made it become crystalline thus facilitates the moisture evaporation at higher temperature [2]. Maximum degradation occurs at temperature of about 367 ${ }^{0} \mathrm{C}-370{ }^{0} \mathrm{C}$ which is mainly expected due to the contribution of cellulose. Cellulose molecule is a very long polymer of glucose units and its crystalline region improved the thermal stability. This supported by how degradation temperature of cellulose shifted to higher temperature for PA and PB. Increase in hydrogen bond between cellulose chains can lead to more organized and packed cellulose region thus help in increasing thermal decomposition of cellulose [1].

Table 1 summarized thermal behaviour of PR, PA and PB. It can be concluded that pre- treatment helps to enhance the thermal stability of PALF thus improved others thermal behaviour of PALF.
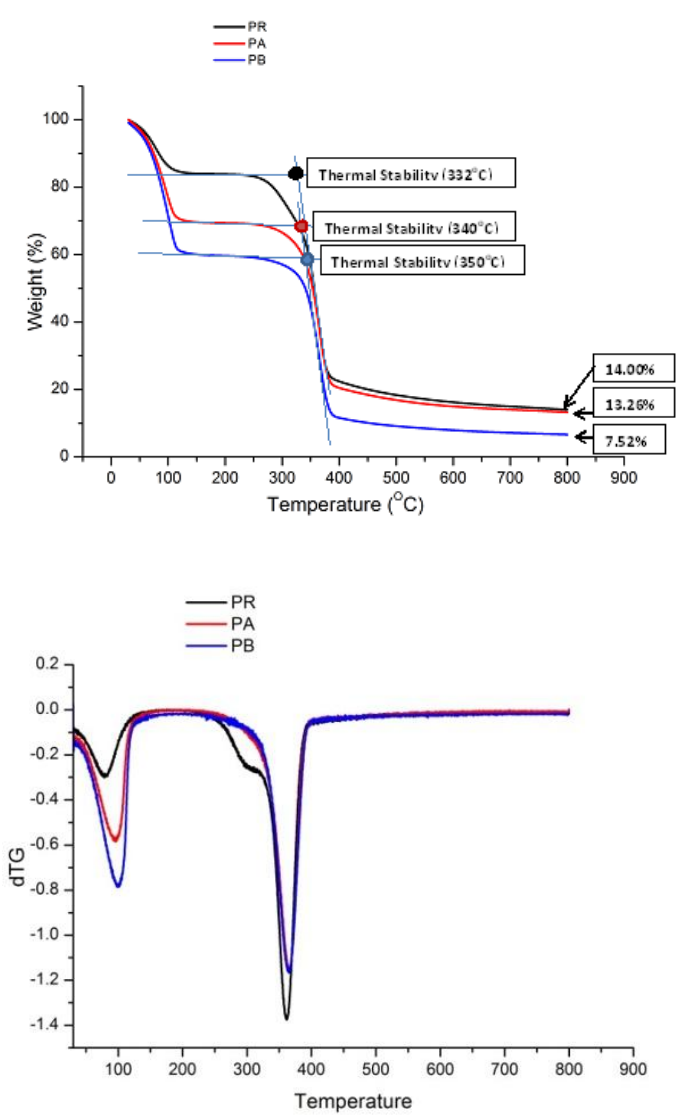

Fig. 4: Thermal graph of PR, PA, PB (a) TGA graph (b) DTG graph

Table 1: Thermal behaviour of raw and variously treated PALF

\begin{tabular}{c|c|c|c|c}
\hline PALF & $\begin{array}{c}\text { Initial Thermal } \\
\text { Stability }\left({ }^{\circ} \mathrm{C}\right)\end{array}$ & $\begin{array}{c}\text { Peak } \\
\text { Tempera- } \\
\text { ture }\left({ }^{\circ} \mathrm{C}\right)\end{array}$ & $\begin{array}{c}\% \text { of the } \\
\text { maximum } \\
\text { degradation }\end{array}$ & $\begin{array}{c}\text { Ash con- } \\
\text { tent at } 800 \\
{ }^{\circ} \mathrm{C}(\%)\end{array}$ \\
\hline PR & 332 & 83 & 47.5 & 14.00 \\
& 340 & 95 & 41.86 & 13.26 \\
PA & & & & \\
PB & 350 & 100 & 41.86 & 7.52 \\
\hline
\end{tabular}

The DSC curves in nitrogen atmosphere of PR, PA and PB were presented in figure 5. The DSC curves for all samples shows 
evaporation of water at temperature $110^{\circ} \mathrm{C}-120^{\circ} \mathrm{C}$ and cellulose degradation at around $330^{\circ} \mathrm{C}-380{ }^{\circ} \mathrm{C}$. Treated PALF exhibits higher entalphy values than untreated PALF. Different chemical processes increase the amount of crystalline cellulose thus increased the H-bonding that resulted in structure stabilized [1].

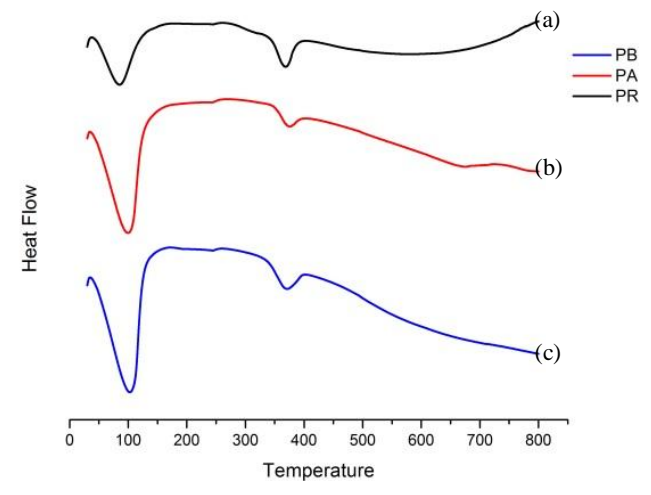

Fig. 5: DSC result of (a) PR (b) PA (c) PB

\subsection{Effect of pre-treatment the morphology of PALF}

Figure above shows surface morphology result for PR and PB samples. PALF contained cylindirical surface that surround bound of assembly microfibrils. Cellulose is organized into fibrils, which are surrounded by matrix of lignin [9] with layer of subtances which may include pectin, lignin, and other impurities [13]. However, alkali and bleaching treatment was believed manage to breakdown the outer surface of PALF fiber (lignin) and hemicelluloses thus produced more separate and singular unifibril PALF such as shown in figure 6 (b).

Figure 6 (c) and 6 (d) shows the SEM result using bigger magnification at $5 \mathrm{~K}$. PB modified the fiber surface thus made it more smooth than those of untreated one due to the hemi, pectin and lignin partially removal. During bleaching treatment, most of the impurities, hemicelluloses and lignin were diminished thus enlighten hidden cellulose fibers to the surface producing crystalline cellulose. On the other half, there were also reduction in the diameter of the fibers from an average of $15 \mu \mathrm{m}$ to $6 \mu \mathrm{m}$. (a)

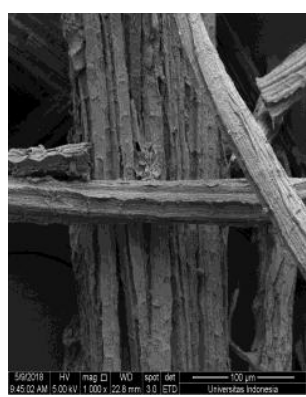

(c)

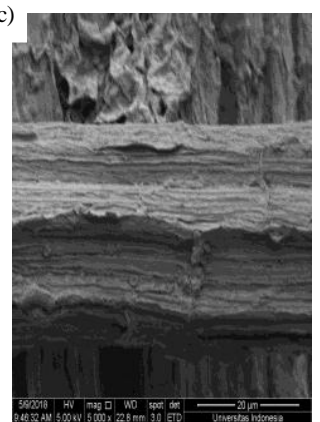

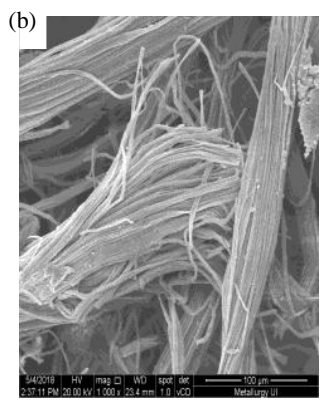

(d)

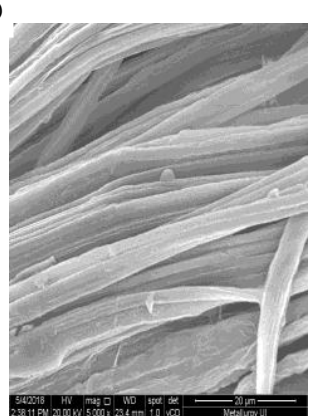

Fig. 6: Surface morphology result of (a) PR (1K x mag), (b) PR (5K x $\mathrm{mag})$ (c) PB (1K x mag) (d) (5K x mag)

\subsection{Effect of pre treatment on chemical structure of electrospun PALF/PET}

Based on few paper written on lignocellulosic fibers [8,9] a peak usually presented at $\sim 1595 \mathrm{~cm}^{-1}$ represent the lignin however were absent at PET/PALF spectrum. This could indicate the reducing amount of lignin in the sample. It is suggested that this is due to the treatment PALF followed by dissolve in TFA that plays role on disrupted the covalent bond that hold the cellulose (lignin) as well eliminates it. Peak at $\sim 1715 \mathrm{~cm}^{-1}$ and $\sim 1099 \mathrm{~cm}^{-1}$ shows a decreasing intensity as shown in figure 3 . This indicated there is interaction between oxygen in the $\mathrm{C}=\mathrm{O}$ and $\mathrm{C}-\mathrm{C}-\mathrm{O}$ group of PET with hydrogen bond of PALF. It is supported by the appearance of broad peak at $3400 \mathrm{~cm}^{-1}$ representing the hydroxyl group resulted from the interaction of PET/PALF contributed to the hydrophilic tendency of the fiber.

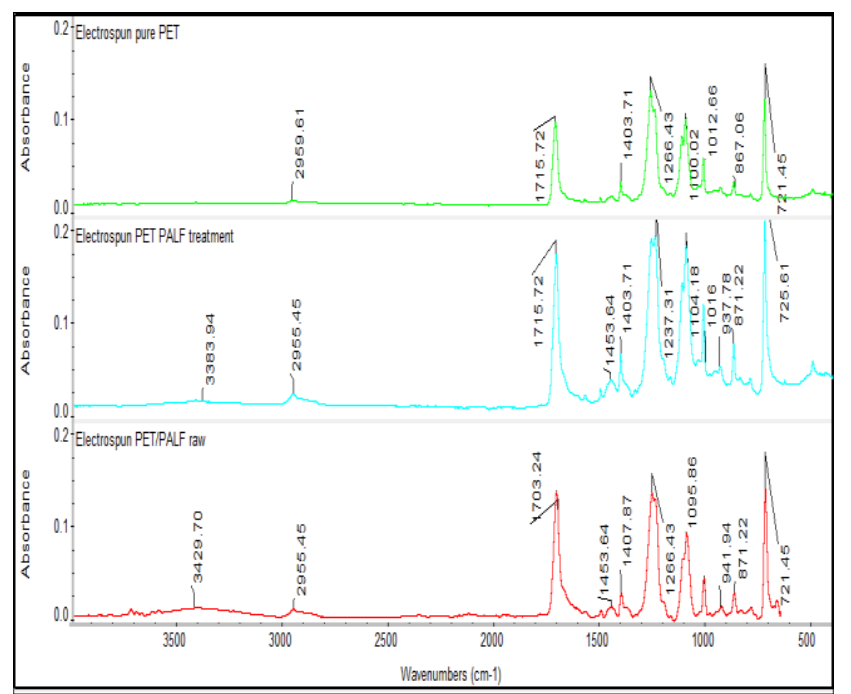

Fig. 7: FTIR result of (a) Electrospun PET, (b) Electrospun PET/PR (c) Electrospun PET/PB

\subsection{Effect of pre-treatment on the morphology of elec- trospun PALF/PET}

FESEM images of electrospun nanofibrous structures of (a) PET and (b) PET/PR and (c) PET/PB shown in Figure 8 revealed that nano-scale of spider web structures were successfully created via electrospinning method. The fibers were found randomly oriented with interconnected pores in between to form a 3D scaffold. Electrospun PET (figure 8(a)) sample shows diameter range between 40-120 nm with average $91.6 \mathrm{~nm}$ while electrospun PET/PR (figure 8(b)) and electrospun PET/PB (figure 8(c)) shows lower fiber average diameter which is $52.1 \mathrm{~nm}$ and $51.6 \mathrm{~nm}$ respectively.

FESEM results indicated that the present of fibers led to a tendency of lower average fiber diameter however has random diameter distribution. Two different fiber networks with intersecting fibers were observed in PET/PALF. It is suggested that one networks with bigger diameter probably corresponds to PET and the others with smaller diameter correspond to PALF. Thicker diameter might due to the increasing solution concentration after adding PALF.

As shows in fig 8 (b), a thin fibers coexited among the electrospun fibers were present. A thin fiber was coexited from single fiber indicated that new fibers were ejected however the bond were collapsed during ejection thus did not produce a complete single fiber. Nevertheless, electrospun PET/PB with treatment shows improvement in the morphology. Smooth single fiber was produced. Fiber without defect could lead to a good mechanical properties of the sample [8]. It may be due to the reducing and removal of lignin, hemicellulose and pectin from PALF that help increase the amount of crystalline cellulose from PALF. 
(a)
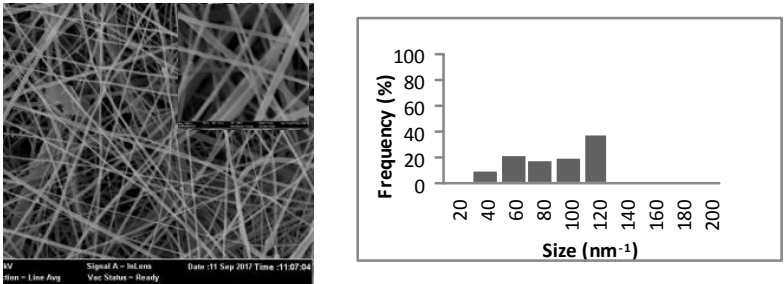

(b)

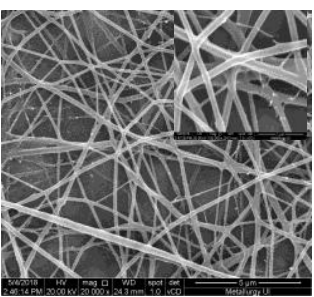

(c)
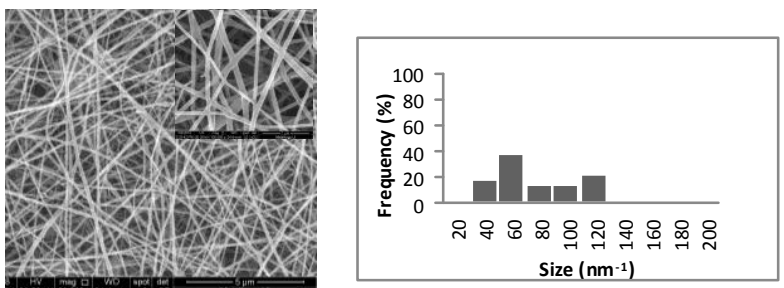

Fig. 8: FESEM result of (a) Electrospun PET, (b) Electrospun PET/PR (c) Electrospun PET/PB

\section{Conclusion}

As a conclusion, FTIR, TGA and DSC studies shows reducing amount of hemicellulose, pectin and lignin thus improve crystalline structure of PALF. FESEM morphology shows decreasing in treated PALF size compared to PR. Thus, pre-treatment PALF favors the formation of smooth fine nanofibers electrospun mats.

\section{Acknowledgement}

The authors would like to acknowledge Universiti Teknologi MARA for providing grant to carry out this research under (600IRMI/MYRA 5/3/GIP (073/2017)

\section{References}

[1] Nadezda S, Viola H \& Adriana E (2016), Study of Thermal Analysis of Selected Cellulose Fibers. GeoScience Engineering 3, 18-21

[2] Deepa B, Eldho A, Bibin MC, Alexander B, Jonny JB, Laly AP, Alcides LL, Sivoney FS \& Kottaisamy M (2011), Structure, morphology and thermal characteristics of banana nano fibers obtained by steam explosion. Bioresource Technology 102,1988-1997

[3] Neni MA, Ida S, Dhewa E, Tri S, Muhammad MM \& Khairurrijal (2017), Polyvinyl Alcohol/Soursop Leaves Extract Composites Nanofibers Synthesized Using Electrospinning Technique and Their Potential as Antibacterial Wound Dressing. Procedia Engineering 170, 31-35

[4] Cintil JC, Lovely M \& Sabu T (2014), Review of recent research in nano cellulose preparation from different lignocellulosic fibers. Rev.Adv.Mater. Sci. 37, 20-28

[5] Neto ARS, Marco AMR, Fernanda VDS, Luiz HCM \& Jose MM (2013), Characterization and comparative evaluation of thermal, structural, chemical, mechanical and morphological properties of six pineapple leaf fiber varieties for use in composites. Industrial Crops and Products 43, 529-537

[6] Manimaran P, Senthamaraikannan P, Sanjay MR, Marichelvam MK \& Mohammad J (2017), Study on Characterization of Furcraea Foetida new natural Fiber as Composite Reinforcement for lightweight Applications. Carbohydrate Polymers https://doi.org/10.1016/j.carbpol.2017.11.099
[7] Sarada PK, Sumit C, Subhasish BM \& Basudam A (2018), Effectiveness of the mild alkali and dilute polymer modification in controlling the durability of jute fibre in alkaline cement medium. Construction and Building Materials 174, 330-342

[8] Bruno VMR, Elaine CR, Rachel POS \& Elisabete F (2015), Ultrathin and nanofibers via room temperature electrospinning from trifluoroacetic acid solutions of untreated lignocellulosic sisal fiber or sisal pulp. Journal of Applied Polymer Science DOI: 10.1002/APP.41826

[9] Rachel POS, Bruno VMR, Elaine CR, Adhemar CRF \& Elisabete F (2015), Bio-based materials from the electrospinning of lignocellulosic sisal fibers and recycled PET. Industrial Crops and Products 72, 69-76

[10] Guihe L, Yuming Z, Mengqing LV, Yan S \& Ding C (2013), Super hydrophilic poly(ethylene terephthalate) (PET)/poly(vinylalcohol) (PVA) composite fibrous mats with improved mechanical properties prepared via electrospinning process. Colloids and Surfaces A: Physicochem. Eng. Aspects 436, 417-424

[11] Guoroi J, Molamma PP, Dan K, Sathesh KA, Kantha DA \& Seeram $\mathrm{R}$ (2013), Tissue engineered plant extracts as nanofibrous wound dressing. Biomaterials 34, 724-734

[12] Santosha PVRK ,Shiva SGA and Manikanth V (2018), Effect of fiber loading on Thermal properties of Banana and Pineapple leaf fiber Reinforced Polyester Composites. Materials Today: Proceedings 5, 5631-5635

[13] Jie C, Rui Z, Tiantian L, Jingren H, \& Guozhen W (2018), Bamboo cellulose-derived cellulose acetate for electrospun nanofibers: synthesis, characterization and kinetics. Cellulose https://doi.org/10.1007/s10570-017-1604-3

[14] Muhammad KBB \& Elammaran J (2016), Comparative study of functional groups in natural fibers: fourier transform infrared analysis (ftir). International Conference on Futuristic Trends in Engineering, Science, Humanities, and Technology ISBN: 978-9385225-55-0

[15] Ismail MF, Nur AI, Wan HWJ, Nur AMI, Ainil HJ \& Fauziah AA (2018), Characteristics of cellulose extracted from Josapine pineapple leaf fibre after alkali treatment followed by extensive bleaching. Cellulose, https://doi.org/10.1007/s10570-018-1878-0

[16] Asim M, Jawaid M, Khalina A \& Mohamad RI (2016), Effect of Alkali and Silane Treatments on Mechanical and Fibre-matrix Bond Strength of Kenaf and Pineapple Leaf Fibres. Journal of Bionic Engineering 13, 426-435

[17] Marianna LT, David S, Claire P, Jean PB, Agnes S, Rene G, Vincent G \& Pierre K (2008), Influence of various chemical treatments on the composition and structure of hemp fibres. Composites: Part A 39, 514-522

[18] Adrianna RM, Maria AM, Odilon RRF \& Luiz HCM (2010), Studies on the thermal properties of sisal fiber and its constituents. Thermochimica Acta 506, 14-19 